Classification

Physics Abstracts

$61.40 \mathrm{M}-61.80 \mathrm{~F}$

\title{
Electron-beam irradiation effects in Al-Ni-Co decagonal quasicrystal
}

\author{
K. Edagawa, K. Suzuki, M. Ichihara and S. Takeuchi
}

Institute for Solid State Physics, The University of Tokyo, Roppongi, Minato-ku, Tokyo 106, Japan

(Received 22 January 1993, accepted 8 March 1993)

\begin{abstract}
By observing the change of the diffraction pattern and the lattice image, structural changes by electron beam irradiation have been investigated for Al-Ni-Co ordered decagonal quasicrystal, which was recently identified by the present authors. By $100 \mathrm{kV}$ electron beam irradiation, essentially no change was detected in the diffraction pattern even after half an hour exposure to the electron beam. On the other hand, by $300 \mathrm{kV}$ irradiation, drastic changes were observed after only a few minutes of irradiation; in the diffraction pattern the diffraction spots became diffuse and all the ordered spots and weak main spots disappeared; in the lattice image, the characteristic ring contrast in the decagonal phase became indistinct. However, no reconstruction of the ring pattern was recognized, suggesting that the phason strains, if introduced, are limited to components of wavelength shorter than the inter-ring distances $(\sim 2 \mathrm{~nm})$.
\end{abstract}

\section{Introduction.}

The decagonal phase is a two-dimensional quasicrystal, the structure of which consists of a period stacking of a quasiperiodic layer having ten-fold symmetry. The decagonal phase was first identified in an Al-Mn alloy by Bendersky [1], soon after the discovery of the first quasicrystal with icosahedral symmetry by Shechtman et al. [2]. Subsequent studies have shown that the decagonal phase is formed in many other Al-based alloy systems: Al-Fe [3], Al-Pd [4], Al-Ni [5], Al-Cu-Co $[6,7], \mathrm{Al}-\mathrm{Ni}-\mathrm{Co}[7]$ and Al-Pd-Mn [8]. The last three alloys have been shown to be thermodynamically stable.

Recently, a new type of decagonal quasicrystal was identified in Al-Ni-Co alloy, which has a superlattice order in the quasiperiodic plane [9]. In this paper we have studied effects of electron beam irradiation on the structure of the ordered Al-Ni-Co decagonal quasicrystal by observing changes of the diffraction pattern and the lattice image. 


\section{Experimental procedures.}

An alloy with the composition of $\mathrm{Al}_{70} \mathrm{Ni}_{17} \mathrm{Co}_{13}$ was prepared from the elemental constituents by arc-melting under an argon atmosphere. The alloy ingot was remelted at $1160{ }^{\circ} \mathrm{C}$ and slowly cooled down to $900^{\circ} \mathrm{C}$ with a cooling rate of $10^{\circ} / \mathrm{h}$ and then furnace-cooled. Columnar grains consisting mainly of the ordered decagonal quasicrystal (and of a small amount of crystal approximants) were taken out by crushing the ingot. They are $3-5 \mathrm{~mm}$ in length and $0.2-0.5 \mathrm{~mm}$ in diameter. Discs with a thickness of $0.4 \mathrm{~mm}$ were cut out perpendicularly to the longitudinal direction of the columnar grains. They were thinned by ion-milling after being polished mechanically. The normal of the discs is approximately parallel to the ten-fold axis of the decagonal quasicrystal.

Electron beam irradiation experiments were performed at room temperature using Hitachi $\mathrm{H}-9000$ transmission electron microscope under the accelerating voltages of $100 \mathrm{kV}$ and $300 \mathrm{kV}$. Structural changes during the electron beam irradiation were studied by electron diffraction for the $100 \mathrm{kV}$ irradiation and by both electron diffraction and lattice image observation for the 300 $\mathrm{kV}$ irradiation.
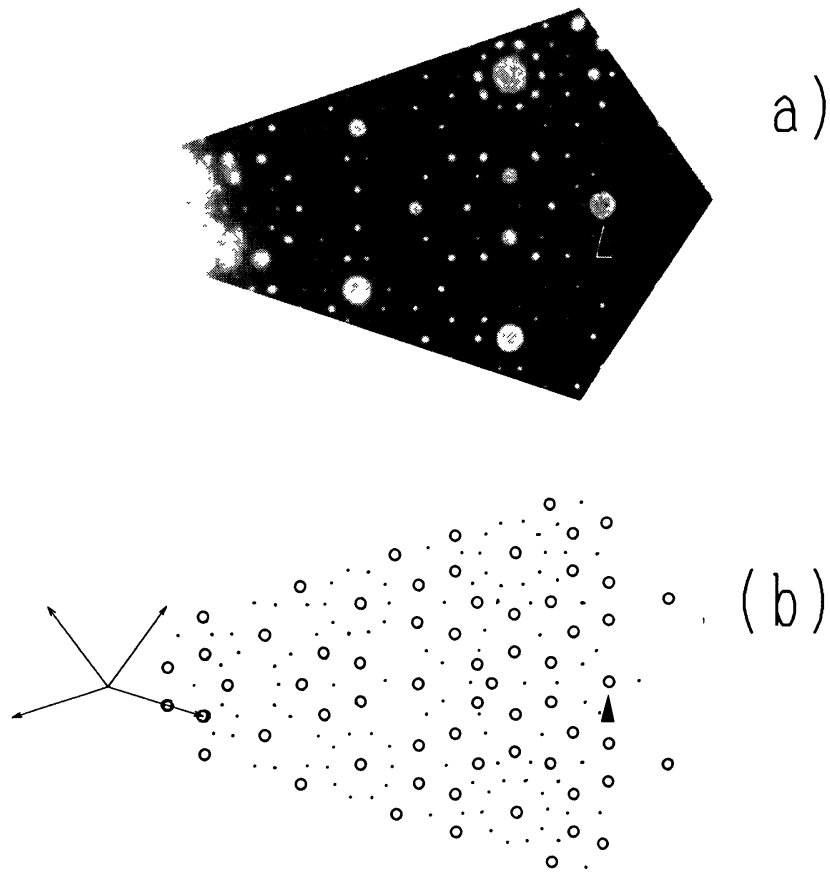

Fig. 1. - A 1/10 section of the ten-fold electron diffraction pattern of the Al-Ni-Co ordered decagonal quasicrystal (a). In (b), classification of diffraction spots in (a) is illustrated: circles and dots denote main spots and ordered ones, respectively. Basis vectors for the main spots are also shown in (b). The spots corresponding to a lattice spacing of about $0.2 \mathrm{~nm}$ are indicated by arrowheads.

\section{Ordered decagonal quasicrystal.}

Figure 1a shows a $1 / 10$ section of the ten-fold electron diffraction pattern from the Al-Ni-Co ordered decagonal quasicrystal. Diffraction spots observed in figure 1a are classified into two 
groups: main spots (circles in figure 1b) and ordered ones (dots). In general, diffraction spots in the ten-fold pattern of decagonal quasicrystals can be indexed by four of the five center-to-vertex vectors of a regular pentagon. If we use the four vectors shown in figure $1 \mathrm{~b}$ as the reciprocal basis vectors, the spots shown by circles can be indexed (with integer indices). On the other hand, those shown by dots can only be indexed with $\left(h_{1} / 5, h_{2} / 5, h_{3} / 5, h_{4} / 5\right)$-type indices. Therefore, the spots shown by circles and those shown by dots in figure $1 \mathrm{~b}$ can be regarded as main and ordered spots, respectively, as in the case of conventional crystals. Detailed discussion on the order-disorder relation has been given elsewhere [9].

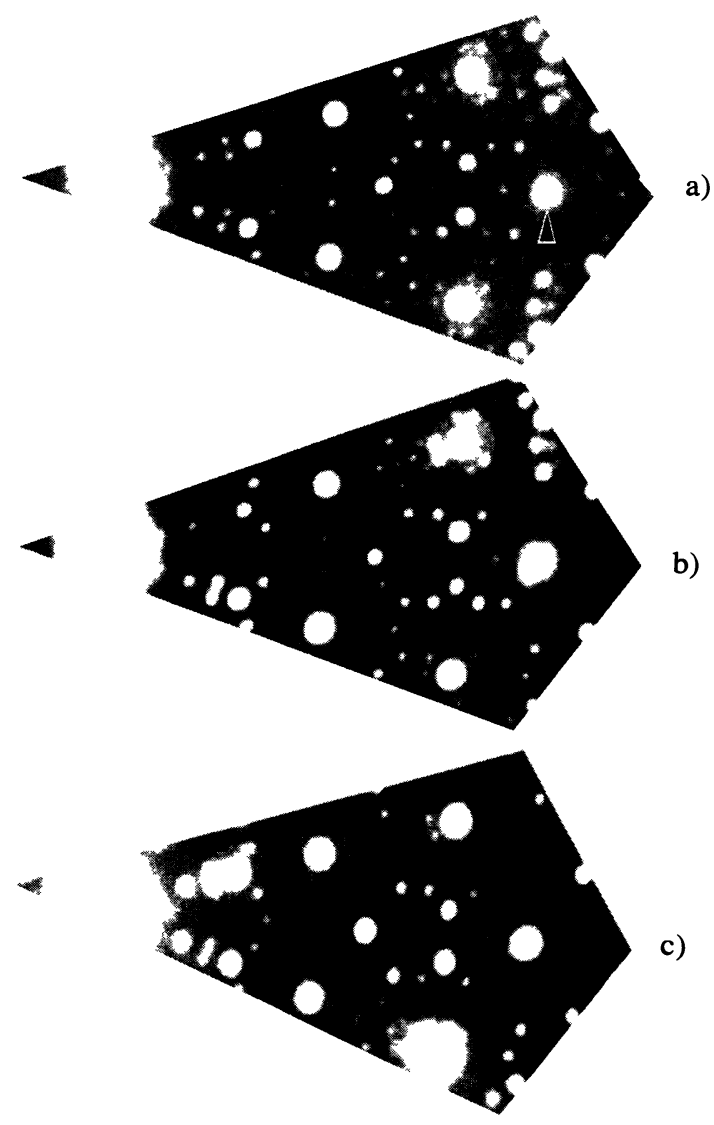

Fig. 2. - The change of ten-fold diffraction pattern during $100 \mathrm{kV}$ electron beam irradiation. Irradiation times are $0 \mathrm{~min}(\mathrm{a}), 10 \mathrm{~min}$ (b) and $30 \mathrm{~min}$ (c). The spot corresponding to a lattice spacing of about $0.2 \mathrm{~nm}$ is indicated by an arrowhead. 


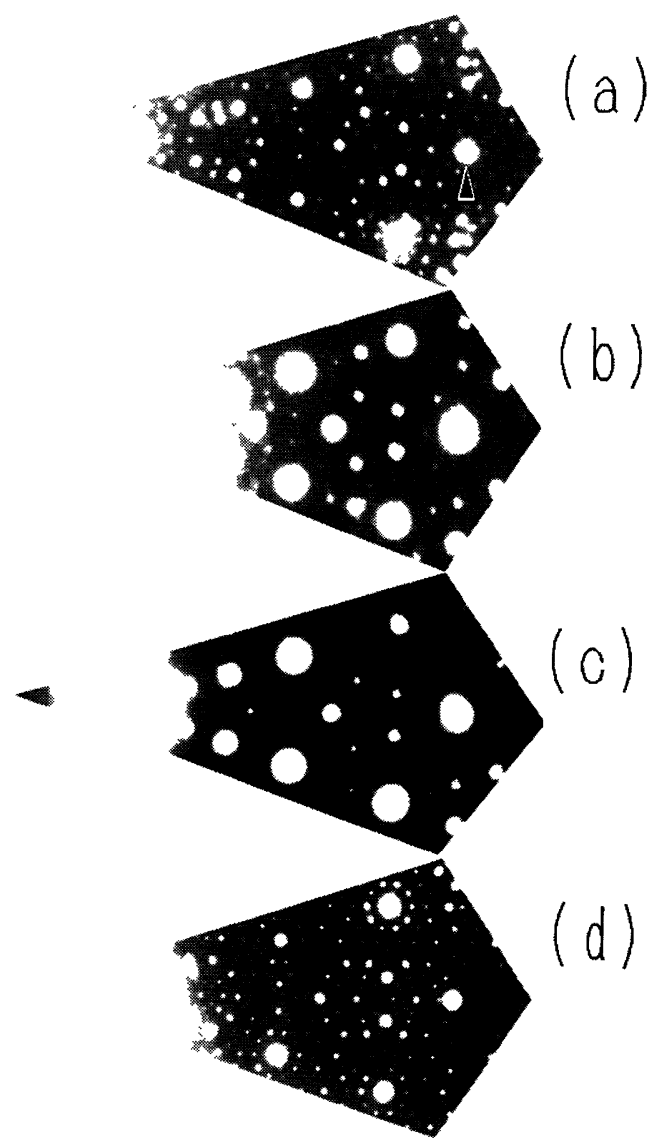

Fig. 3. - The change of ten-fold diffraction pattern during $300 \mathrm{kV}$ electron beam irradiation. Elapsed times are $0 \mathrm{~min}$ (a), $5 \mathrm{~min}$ (b), $10 \mathrm{~min}$ (c) and $10 \mathrm{~min}$ (d). The pattern of (d) was taken from the place $0.2 \mathrm{~nm}$ apart from the original irradiated area, immediately after the pattern of (c) had been taken. The spot corresponding to a lattice spacing of about $0.2 \mathrm{~nm}$ is indicated by an arrowhead.

\section{Experimental results and discussion.}

Figures 2 and 3 show the changes of the ten-fold diffraction pattern during irradiation by electron beam of $100 \mathrm{kV}$ and $300 \mathrm{kV}$, respectively. Many ordered spots are seen in figure 2a and figure $3 \mathrm{a}$, showing that the initial structure is the ordered decagonal phase. By $100 \mathrm{kV}$ electron beam irradiation, no noticeable change is observed. Ordered spots are still observed clearly in the pattern after 30 minutes irradiation (Fig. 2c). In contrast, by $300 \mathrm{kV}$ irradiation, drastic changes are observed. Immediately after the beginning of the irradiation, all spots start to become diffuse; in particular ordered spots begin to disappear. As seen in figure $3 \mathrm{~b}$, most of the ordered spots have already disappeared after five minutes irradiation. After ten minutes irradiation, all the ordered spots have disappeared almost completely, showing that the structure has lost the superlattice order. In figure 3c, not only the ordered spots but also some of weak main spots become invisible. After ten minutes irradiation, we took the electron diffraction pattern from the place apart from 
the original irradiated area by about $0.2 \mathrm{~nm}$ (Fig. 3d). It exhibits many ordered spots clearly, indicating that the changes of the diffraction pattern from figure $3 \mathrm{a}$ to $3 \mathrm{c}$ were not caused by heat induced by irradiation but by irradiation itself.

(a)

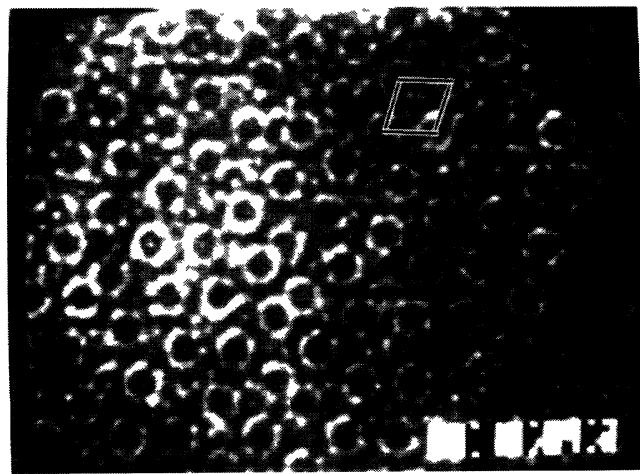

(c)

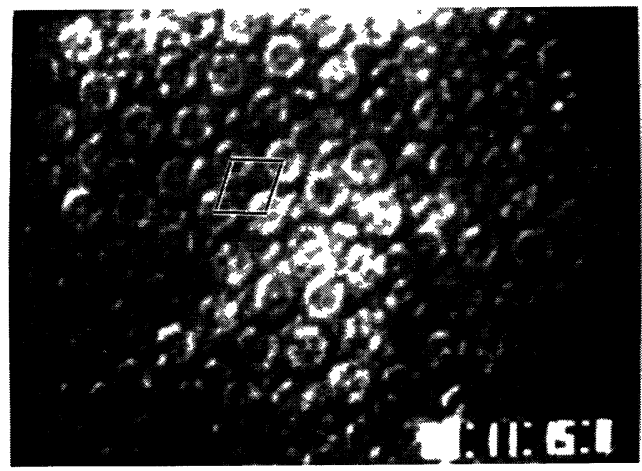

$(b)$

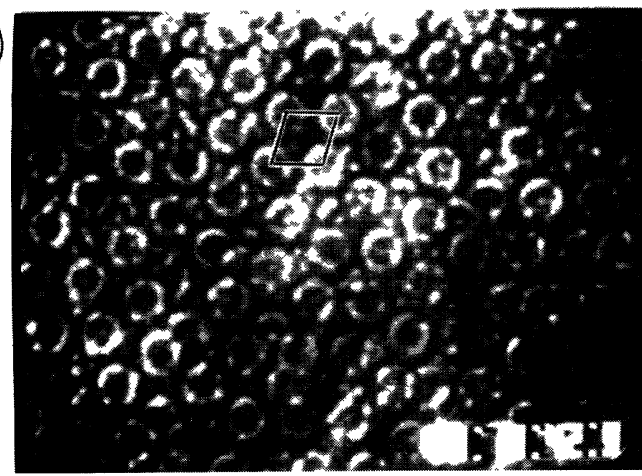

(d)

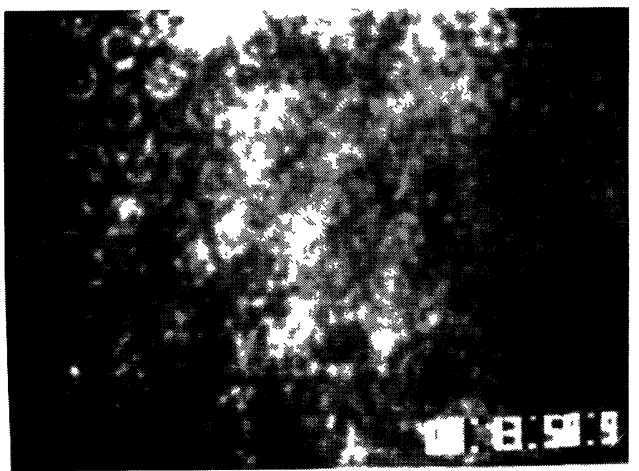

$5 \mathrm{~nm}$

Fig. 4. - The change of lattice image during $300 \mathrm{kV}$ electron beam irradiation. The incident beam is parallel to the ten-fold axis of the decagonal quasicrystal. Irradiation times are $0 \mathrm{~s}(\mathrm{a}), 33 \mathrm{~s}(\mathrm{~b}), 66 \mathrm{~s}$ (c) and $229 \mathrm{~s}(\mathrm{~d})$. One of the corresponding rhombi is drawn in $(\mathrm{a}-\mathrm{c})$ for convenience of comparison.

Figure 4 shows the change of the lattice image during $300 \mathrm{kV}$ electron beam irradiation. In figure 4, the incident beam is parallel to the ten-fold axis of the decagonal quasicrystal. It has been reported by Hiraga [10] that the arrangement of "ring contrasts" is observed in the lattice image of the decagonal phase under a special condition of defocus and sample thickness. The lattice image of the initial structure shown in figure $4 \mathrm{a}$ exhibits clearly those ring contrasts. The ring contrasts become less and less distinct as the irradiation time increases (Figs. 4b and c), and finally they disappear almost completely after about four minutes irradiation (Fig. 4d). However, careful examination reveals that the above-mentioned change of the lattice image is not accompanied by any rearrangement of the ring contrasts (one of the corresponding rhombi is drawn in Figs. 4a-c for convenience of comparison).

Urban et al. have observed the amorphization of Al-Mn icosahedral and decagonal phases and $\mathrm{Al}-\mathrm{V}$ icosahedral phase by electron beam irradiation [11]. They have shown that the quasicrystalline phases transform into nearly complete amorphous structure. This is in contrast to our 
result in which intense reflections remain spotty after the irradiation. The different behavior may be attributable to the difference in the stability of the quasicrystals.

In quasicrystalline materials, a special type of defects called phason strain could be introduced [12]. Actually, $G_{\perp}$ (phason momentum) -dependence of X-ray diffraction peak widths [13-15] or shifts of bright-dot rows in high resolution electron micrograph $[16,17]$, which are attributed to phason strain, are often observed for quasicrystalline phases. It is, therefore, plausible to consider that the structural changes observed in the present experiments are caused by the introduction of the phason strain. It should be noted, however, that the phason strain, if introduced, must be limited to components of wavelength shorter than the inter-ring distances, because long wavelength components would induce the rearrangement of the ring contrasts.

Tei et al. [18] have simulated in computer amorphizatin of an icosahedral quasicrystalline structure through chemical disordering process. They have obtained successfully a model binary amorphous phase by introducing chemical disorder (i.e. by exchanging the two elements randomly) into a model binary icosahedral quasicrystal, followed by relaxation using interatomic potentials. This process, in addition to the introduction of the above-mentioned phason strain, may cause the structural changes observed in the present experiments.

\section{References}

[1] BENDERSKY L., Phys. Rev. Lett 55 (1985) 1461.

[2] Shechtman D., Blech I., Gratias D. and Cahn J.W., Phys. Rev. Lett. 53 (1984) 1951.

[3] FunG K.K., YANG C.Y., ZHOU Y.Q., ZHAO J.G., ZHAN W.S. and SHEN B.G., Phys. Rev. Lett. 56 (1986) 1915.

[4] IDZIAK S., HEINEY P.A. and BANCEL P.A., Mater. Sci. Forum 22-24 (1987) 353.

[5] Li X.Z.Z. and Kuo K.H., Philos. Mag. Lett. 58 (1988) 167.

[6] HE L.X., WU Y.K. and KuO K.H., J. Mater. Sci. Lett. 7 (1988) 1284.

[7] TSAi A.P., INOUE A. and MASUMOTO T., Mater. Trans. JIM 30 (1989) 463.

[8] BeEli C., NisSEN H.U. and Robadey J., Philos. Mag. Lett. 63 (1991) 87.

[9] Edagawa K., Ichihara M., SuZuki K. and TAKeuCHI S., Philos. Mag. Lett. 66 (1992) 19.

[10] HiRAGA K., "Quasicrystals: The State of the Art", P.J. Steinhardt and D. Di Vicenzo Eds. (Singapore: World Scientific, 1991).

[11] Urban K., MAYer J., RAPP M., Wilkens M., Csanady A. and Fidler J., J. Phys. 47 (1986) C3-465.

[12] Socolar J.E.S., LubENSKY T.C. and STEINHARDT P.J., Phys. Rev. B 34 (1986) 3345.

[13] HoRn P.M., MALZFELDT W., Di VinCEnZo K.P., TONER J. and GAMBIno R., Phys. Rev. Lett. 57 (1986) 1444.

[14] Heiney P.A., Bancel P.A., HoRn P.M., Jordan J.L., Laplaca S., Angilello J. and Gayle F.W., Science 238 (1987) 660.

[15] EdaGAWA K., Philos. Mag. Lett. 61 (1990) 107.

[16] HIRAGA K. and HiRABAYASHI M., J. Electron Microsc. 36 (1987) 353.

[17] Li F.H., PAN G.Z., HUANG D.X., HASHIMOTO H. and YoKOTA Y., Ultramicroscopy 45 (1992) 299.

[18] Tei T., Takeuchi S. and Kimura K., J. Non-Cryst. Solids 117/118 (1990) 769. 